\title{
New nuclear structure data beyond ${ }^{132} \mathrm{Sn}$
}

\author{
Radomira Lozeva ${ }^{1,2, *}$ \\ ${ }^{1}$ CSNSM, CNRS/IN2P3, Université Paris-Saclay, 91405 Orsay Campus, France \\ ${ }^{2}$ IPHC, CNRS/IN2P3, Université de Strasbourg, 67037 Strasbourg, Cedex 2, France
}

\begin{abstract}
Exotic nuclei beyond the ${ }^{132} \mathrm{Sn}$ double shell-closure are influenced by both the Sn superfluity and the evolving collectivity only few nucleons away. Toward even more neutron-rich nuclei, especially at intermediate mass number, the interplay between single-particle and collective particle-hole excitations competes. In some cases with the extreme addition of neutrons also other effects as the formation of neutron skin, stabilization as sub-shell gaps or orbital crossings may be expected. The knowledge of nuclear ingredients is especially interesting beyond ${ }^{132} \mathrm{Sn}$ and little is known on how the excitation modes develop with the addition of both protons and neutrons and for example systematic prompt and decay studies can be such very sensitive probe. Recently, we have approached this region of nuclei in several experimental measurements following ${ }^{238} \mathrm{U}$ projectile fission on ${ }^{9} \mathrm{Be}$ and $\mathrm{n}$-induced fission on ${ }^{241} \mathrm{Pu}$ and ${ }^{235} \mathrm{U}$. Consistent data analysis allows to access various spins and excitation energies and provide new input to theory. Examples from these studies on several nuclei in the A 140 region were presented during the conference together with the possible interpretation of the new data. Here, we will illustrate one example on ${ }^{136}$ I using two complementary data sets.
\end{abstract}

\section{Introduction and Motivation}

Nuclei beyond ${ }^{132} \mathrm{Sn}$ present very particular properties. On one hand they are exotic, being far from stability, on the other - simple, as they are located close to the doubly-magic spherical nucleus ${ }^{132} \mathrm{Sn}$. Thus, generally single-valence particle excitations of few neutrons as in ${ }^{135} \mathrm{Sb},{ }^{136} \mathrm{Sb}[1,2]$ up to few protons away as in ${ }^{135} \mathrm{I},{ }^{136} \mathrm{I}$ $[3,4]$ present rather simple descriptions. An onset of collectivity is expected for these nuclei as soon as both protons and neutrons are added onto the core system. Currently available experimental data compared to theory shows that e.g. ${ }^{138} \mathrm{Te}$ [5] is at the border to develop some deformation [6]. Experimental excitation energies, compared to theory report on quadrupole and possible octupole collectivity above Xe, toward the heavy Ba region $[7,6]$ despite expectations in ${ }^{139} \mathrm{Te}[8,9]$.

To date, only a few shell-model descriptions are available in this region, where ${ }^{132} \mathrm{Sn}$ is treated as a core and few-valence particle excitations in the lower-lying $v f_{7 / 2}, \quad v p_{3 / 2}$ and $\pi g_{7 / 2}, \pi d_{5 / 2}$ reasonably describe the beginning $[8,10]$ of these shells. In addition, some polarisation of the core has to be assumed. More monopole corrections are needed e.g. at the end of the $v f_{7 / 2}$ shell, around $N=90$, to describe our recent experimental data on possible crossing between $\pi g_{7 / 2}$ and $\pi d_{5 / 2}$ orbitals [11]. Even if some of these descriptions do reasonably well close to ${ }^{132} \mathrm{Sn}$, they show discrepancies e.g. in the position of the generally isolated $\pi g_{7 / 2}$ and $\pi d_{5 / 2}$ orbitals. In particular, this relates to the calculated depressed position of $\pi d_{5 / 2}$ for ${ }^{136} \mathrm{I},{ }^{138} \mathrm{I}[8,12]$ believed to be responsible for their compressed low-energy spectra, resulting in the existence of long-lived isomers [13]. On the neutron side, differences exist in the predictions for $N=90$ sub-shell gap between the $v f_{7 / 2}$ and $v p_{3 / 2}$ orbitals [14], an assumption inconsistent with [15]. Having worked in this region in several works to date, we provide indispensable ingredients to understand many of the open questions. For this, it is important to further investigate specifically the species close to $N=82$, to compare them with and explore the entire proton-neutron particle valence space beyond.

\section{Measuremens and Data}

Several experimental investigations were recently performed using various methods, as on the very neutron-rich nuclei - in isomer and $\beta$-decay spectroscopy at EURICA, the less neutron-rich in prompt $\gamma$-ray spectroscopy at EXILL and in purely $\beta$-decay measurements at LOHENGRIN. Details of these studies were discussed elsewhere $[2,5,11,16,17]$. The strategy in these complementary measurements is to find a common description of the entire region on orbital evolution and collectivity, while searching for single-particle and collective excitations and their persistence with the addition of particles. Here, we will give one example on the observation of high-energy low spin states for $Z=53$ nuclei, that we traced towards the end of the $v f_{7 / 2}$ shell at ${ }^{140} \mathbf{I}_{87}$ [18] and at its beginning at ${ }^{136} \mathbf{I}_{83}$ [17]. In the ${ }^{140} \mathbf{I}$

- Corresponding author: radomira.lozeva@csnsm.in2p3.fr 
case, the states were populated in pure $\beta$-decay of Te projectiles, while for ${ }^{136} \mathrm{I}$, they were populated in the prompt data of fission fragments, which accessed also $\beta$ decay states in the daughter nucleus, as well as in another $\beta$-decay experiment of $\mathrm{Te} \rightarrow \mathrm{I}$ nuclei.

Particular attention for these iodine nuclei shall be drawn to the excitations taking place at high energy and lowspin for the $\left(1^{+}\right)$states, which are changing parity with respect to the negative parity g.s. They are interesting because they represent changing configurations, different from those in $\mathrm{Sb}$ and $\mathrm{Te}$ isotopes. Appearing between $v h_{9 / 2} \rightarrow \pi h_{11 / 2}$ orbitals, which are open at $Z=53$ for the iodine nuclei, below $Z=52$ these allowed-type transitions are completely blocked [19].

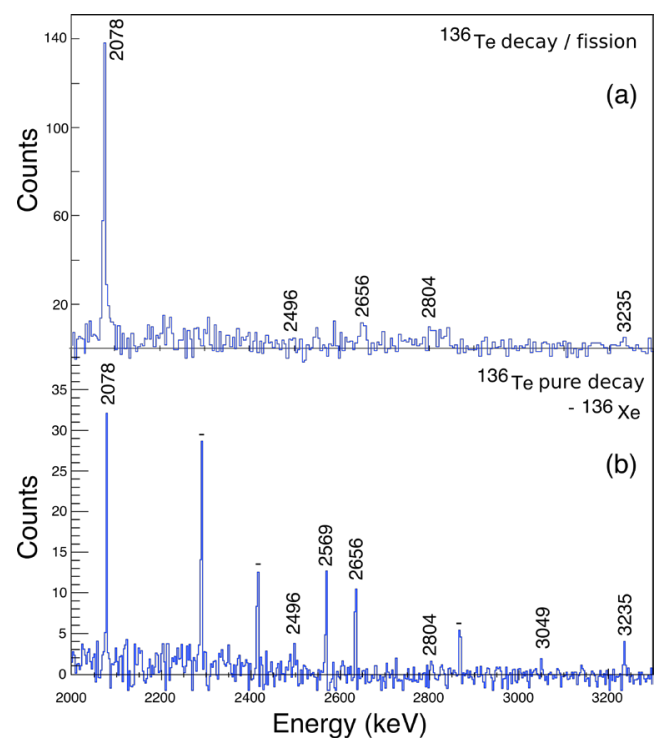

Fig. 1. Decay $\gamma$-ray transitions from $1^{+}$levels in ${ }^{136}$ I observed in $\beta$-decay of products of prompt fission data (a) and in pure ${ }^{136} \mathrm{Te} \beta$-decay (b).

This leads to possible structures of type $\left[\left(v h_{9 / 2}\right)\left(\pi h_{11 / 2}\right)\left(\pi g_{9 / 2}\right)^{2}{ }_{0+}\right]_{1+}$ and $\left[\left(v f_{7 / 2}\right)\left(\pi h_{11 / 2}\right)\left(\pi g_{7 / 2}\right)^{2}{ }_{2+}\right]_{1+}$ [20]. For ${ }^{136} \mathrm{I}$ this was suggested to be due possible configuration mixing of the $\left(v h_{9 / 2}\right)^{2}$ and $\left(v f_{7 / 2}\right)\left(v h_{9 / 2}\right)$ in the g.s. of ${ }^{136} \mathrm{Te}$. Three such candidate states were than proposed in the $\beta$-decay level scheme at relatively high excitation energy of $2656.4,3137.1$ and $3235.2 \mathrm{keV}$. They were identified by evaluators as $1^{+}$states [13], although no firm justification has been experimentally presented except for the first excited $1^{+}$state (at 2656.4 $\mathrm{keV}$ ) [20]. The previously observed $\gamma$-ray transitions depopulating these levels in ${ }^{136}$ I represent the main decay path of the mother nucleus to the $1^{-}$g.s. through either direct high-energy transitions or through the population of intermediate negative-parity levels. It is interesting to note here, that two of these $1^{+}$candidate states at 2656.4 and $3235.2 \mathrm{keV}$ have rather strong decay branches, while the third candidate placed in between (at $3137.1 \mathrm{keV}$ ) has a largely suppressed intensity of its decay transitions. It may thus be possible that this state has a different assignment e.g. leaving only two $1^{+}$states present in ${ }^{136} \mathrm{I}$. This is supported also by the observed $I_{\beta}$ and $\log f t$ values.

We populated these states in prompt data following the n-induced fission of ${ }^{241} \mathrm{Pu}$, where non-yrast $\beta$-decaying states could be accessed. These transitions are detected in a coincidence gate with the lowest transitions $(87+135$ $\mathrm{keV}$ ) in ${ }^{136} \mathrm{I}$, de-exciting its first and second excited states. The resulting spectrum is presented in Fig.1(a). Independently, in a purely $\beta$-decay experiment, we have identified the ${ }^{136} \mathrm{Te}$ nucleus and collected data, corresponding to its decay to ${ }^{136} \mathrm{I}$ with appropriate $\mathrm{T}_{1 / 2}$. The resulting relevant spectrum is presented in Fig.1. (b), where all previously known transitions in ${ }^{136} \mathrm{I}$ are shown. In addition, we have identified transitions from the grand-daughter ${ }^{136} \mathrm{Xe}$, present in the time gate.

\section{Discussion}

Except the strong decay from the first $1^{+}$state by the $2077.9 \mathrm{keV} \gamma$-ray, it is interesting to underline the detection of a $2656.0 \mathrm{keV}$ transition, expected [13], but unobserved as a direct decay from this first $1^{+}$state to the $1^{-}$g.s. state. It appears in the gated spectrum in Fig.1(a), therefore it is a transition depopulating another state at e.g. $3235.2 \mathrm{keV}$, proposed in [20]. The $3235.2 \mathrm{keV}$ transition, proposed as a similar direct branch decay to the g.s. by [20] is also present in this particular gated spectrum. The relative intensities between the transitions within the same data set may be compared. For this, one can neglect the different type of population between them for these levels, which will be discussed elsewhere together other new spectroscopy data on ${ }^{136} \mathrm{I}[21]$.

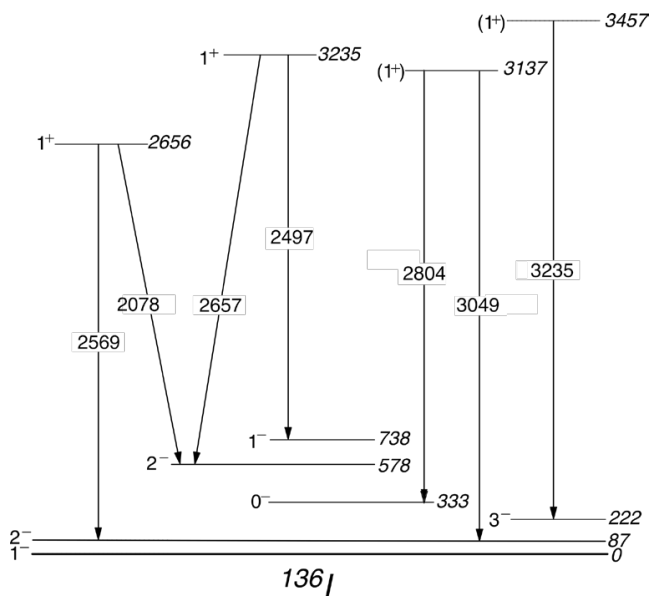

Fig. 2. Partial level scheme of ${ }^{136}$ I with the relevant $1^{+}$states.

Related to the current discussion, it is interesting to point out, that one may possibly observe the $2656.0 \mathrm{keV}$ transition with the same energy decaying out of the third 
$1^{+}$level, while the $3235.2 \mathrm{keV}$ transition may represent depopulation of another fourth $1^{+}$level in ${ }^{136} \mathrm{I}$. The intensities of the transitions de-exciting the second $1^{+}$ level (at $3137.1 \mathrm{keV}$ ) of $2804.0 \mathrm{keV}$ and a new fourth $1^{+}$ level (at $3457.3 \mathrm{keV}$ ) are thus comparable. Partial level scheme of ${ }^{136} \mathrm{I}[13,20]$ with decay branches from all possible $1^{+}$levels is presented in Fig. 2 .

Furthermore, the observation of three $1^{+}$candidates in ${ }^{140}$ I [18], support these assignments. According to the observed $I_{\beta}$ and logft values indeed the first and third $\left(1^{+}\right)$ levels are populated stronger than the second $\left(1^{+}\right)$ candidate state. This points at the a different origin of the second $1^{+}$state, similarly to ${ }^{136} \mathrm{I}$. The population of allowed transitions is observed by the strong feeding of first and third $1^{+}$states in both isotopes, which are suppressed in energy with the increase of $N$, consistent with [18].

\section{Future plans}

The newly obtained data, related to new spectroscopy in ${ }^{136} \mathrm{I}$, as well as other I nuclei in the region are subject of dedicated articles.

\section{References}

1. A. Korgul et al, Phys. Rev. C 91, 027303 (2015)

2. R. Lozeva et al, Phys. Rev. C 92, 024304 (2015)

3. P. Spagoletti et al, Phys. Rev. C 95, 021302 (2017)

4. W. Urban et al, Eur. Phys. Journ. A 27, 257 (2006)

5. P. Lee at al, Phys. Rev. C 92, 044320 (2015)

6. H. Naidja et al, Phys. Rev. C 96, 034312 (2017)

7. D. Bianco et al, Phys. Rev. C 88, 024303 (2013)

8. W. Urban et al, Phys. Rev. C 65, 024307 (2002)

9. R. Kshetri et al, Phys. Rev. C 74, 034314 (2006)

10. L. Corraggio et al, Phys. Rev. C 80, 021305(R) (2009)

11. R. Lozeva et al, Phys. Rev. C 93, 014316 (2016)

12. T. Rzaca-Urban et al, Phys. Rev. C 75, 054319 (2007)

13. www.nndc.bnl.gov

14. S. Sarkar et al, Phys. Rev. C 78, 024308 (2008)

15. H. Naidja et al, Journ. Phys. 580, 012030 (2015)

16. M. Jentschel et al, Journ. Instr. 12, P11003 (2017)

17. R. Lozeva et al, Eur. Phys. Journ., in press (2017)

18. B. Moon et al, Phys. Rev. C 95, 044322 (2017)

19. I. Borzov et al, Phys. Rev. C 67, 025802 (2003)

20. F. Schussler et al, Z. Phys. A 283, 43 (1977)

21. R. Lozeva et al, Phys. Rev. C (to be published) 